'Unidad de Coloproctología, Departamento de Cirugía Digestiva, Pontificia Universidad Católica de Chile. Santiago, Chile. Unidad de Nutrición, Departamento de Cirugía Digestiva, Pontificia Universidad Católica de Chile. Santiago, Chile. ${ }^{3}$ Residente Unidad de Coloproctología, Departamento de Cirugía Digestiva, Pontificia Universidad Católica de Chile. Santiago, Chile.

${ }^{4}$ Unidad de Cirugía Hepatobiliopancreática y Trasplante, Departamento de Cirugía Digestiva, Pontificia Universidad Católica de Chile. Santiago, Chile.

Sin necesidad de apoyo financiero ni conflictos de intereses.

Recibido el 8 de abril de 2016, aceptado el 18 de octubre de 2016.

Correspondencia a: Dr. Javier Gómez Basauri Marcoleta 350, patio interior, Santiago, Chile. javierigomez@gmail.com

\section{Insuficiencia intestinal secundaria a síndrome de intestino corto: resultados de un programa multidisciplinario de rehabilitación intestinal}

\author{
MARÍA ELENA MOLINA ${ }^{1}$, FELIPE BELLOLIO ${ }^{1}$, \\ JULIETA KLAASSEN², JAVIER GÓMEZ ${ }^{3}$, CONSTANZA VILLALÓN ${ }^{3}$, \\ JUAN FRANCISCO GUERRA ${ }^{4}$, ÁLVARO ZÚNIIGA ${ }^{1}$
}

\section{Intestinal failure due to short bowel syndrome: impact of a multidisciplinary intestinal rehabilitation program}

Background: In patients suffering intestinal failure due to short bowel, the goal of an Intestinal Rehabilitation Program is to optimize and tailor all aspects of clinical management, and eventually, wean patients off lifelong parenteral nutrition. Aim: To report the results of our program in patients suffering intestinal failure. Patients and Methods: A registry of all patients referred to the Intestinal Failure unit between January 2009 and December 2015 was constructed. Initial work up included prior intestinal surgery, blood tests, endoscopic and imaging studies. Also demographic data, medical and surgical management as well as clinical follow-up, were registered. Results: Data from 14 consecutive patients aged 26 to 84 years (13 women) was reviewed. Mean length of remnant small bowel was $100 \mathrm{~cm}$ and they were on parenteral nutrition for a median of eight months. Seven of 14 patients had short bowel secondary to mesenteric vascular events (embolism/thrombosis). Medical management and autologous reconstruction of the bowel included jejuno-colic anastomosis in six, enterorraphies in three, entero-rectal anastomosis in two, lengthening procedures in two, ileo-colic anastomosis in one and reversal Roux-Y gastric bypass in one. Thirteen of 14 patients were weaned off parenteral nutrition. Conclusions: Our Multidisciplinary Intestinal Rehabilitation Program, allowed weaning most of the studied patients off parenteral nutrition.

(Rev Med Chile 2016; 144: 1410-1416)

Key words: Anastomosis, Surgical; Intestinal Pseudo-Obstruction; Parenteral Nutrition; Short Bowel Syndrome.
L a insuficiencia intestinal (II) en adultos, definida como la incapacidad de absorción de nutrientes, agua y electrolitos necesarios para el funcionamiento del organismo, puede ser secundaria a resecciones intestinales que determinan un intestino corto, a ostomías, fístulas proximales en el intestino delgado (ID) que impiden la función del intestino distal, daño actínico o a algún trastorno funcional del ID por enfermedades crónicas como la pseudo-obstrucción intestinal crónica ${ }^{1-3}$. No existe consenso para una definición anatómica de intestino corto, siendo este umbral distinto para diferentes autores. Algunos, lo definen cuando la longitud del intestino remanente es menor a $200 \mathrm{~cm}$, otros, si es menor a $150 \mathrm{~cm}^{1,3-5}$. Si esta longitud intestinal resulta insuficiente para 
la adecuada absorción de nutrientes, vitaminas y minerales se manifestará clínicamente como un síndrome de intestino corto o $\mathrm{SIC}^{3-5}$. Dentro de las causas más frecuentes destaca la enfermedad isquémica secundaria a eventos vasculares mesentéricos (trombosis/embolías), la enteropatía isquémica de bajo flujo, la Enfermedad de Crohn, complicaciones quirúrgicas de cirugías intestinales y daño actínico ${ }^{2,3,6}$.

La adaptación intestinal (AI) es un mecanismo de respuesta a la resección de extensos segmentos de ID y tiene como objetivo restaurar la normal absorción de macronutrientes, agua y minerales. Se encuentra siempre presente, iniciándose en el postoperatorio inmediato y puede demorar incluso años en consolidarse. Este proceso ocurre cuando el ID remanente se acerca a la normalidad en términos anatómicos y funcionales ${ }^{7-10}$. La nutrición parenteral (NP) permite la sobrevida del paciente durante el proceso de AI, pero si ésta no logra revertir la II, pasa a ser indispensable y permanente. La NP prolongada se puede acompañar de graves complicaciones como la infección o trombosis de accesos venosos centrales, daño hepático no alcohólico (NAFLD) y compromiso de la calidad de vida. El riesgo de mortalidad por complicaciones derivadas de la NP en pacientes no oncológicos alcanza el 10-15\% cuando se prolonga sobre 2 años ${ }^{11,12}$. En este escenario es prudente centrarse en optimizar y maximizar la función del intestino remanente para intentar suspender la NP y en los casos en que este proceso fracase, considerar el trasplante intestinal. Para cumplir este objetivo es necesario coordinar medidas terapéuticas que incluyen: NP, tratamiento de enfermedades concomitantes y/o de la sepsis abdominal crónica (abscesos, colecciones y fístulas entero-entéricas o entero-cutáneas), manejo dietético y farmacológico del intestino, tratamiento de la proliferación bacteriana, reconstitución quirúrgica autóloga del intestino y elongación del ID remanente si fuese necesario. Todo este manejo debe ser realizado por un equipo multidisciplinario de alto nivel de especialización e interés en esta patología, siendo este uno de los pilares en el éxito de los pacientes evaluados por cada programa ${ }^{10}$.

La referencia de pacientes con II al Hospital Clínico de la Universidad Católica de Chile hizo que en el año 2009 los Departamentos de Cirugía Digestiva, Nutrición y Gastroenterología crearan la Unidad de Insuficiencia Intestinal. Esta Unidad reúne y coordina cirujanos digestivos, nutriólogos, nutricionistas, gastroenterólogos y enfermeras especializadas en ostomías, con el fin de maximizar el rendimiento enteral e intentar la suspensión en forma permanente de la NP, a través de un proceso de rehabilitación intestinal $(\mathrm{RHI})^{13,14}$. Actualmente este grupo ofrece todas las alternativas de manejo de la II, desde el tratamiento general hasta el trasplante intestinal, tanto en pacientes adultos como pediátricos ${ }^{10}$.

El objetivo de esta comunicación es dar a conocer los resultados de un grupo de pacientes con II en NP sometidos a un Programa de RHI.

\section{Material y Método}

Estudio descriptivo y retrospectivo. Se incluyó a todos los pacientes referidos a la Unidad de Insuficiencia Intestinal entre enero de 2009 y diciembre de 2015. Se construyó un archivo en donde se registraron los datos demográficos, causa de la II, longitud del ID y colon remanentes, tiempo de NP total (NPT) previo ingreso al Programa de RHI y variables nutricionales. Cabe señalar que la mayoría de los pacientes que entraron al programa lo hicieron en NPT y provenían de nuestra Red de Salud o derivados desde diferentes centros públicos y privados del Sistema Nacional de Salud. Se practicaron exámenes hematológicos y endoscópicos, además de enteroclisis por tomografía axial computada (TAC) para conocer la anatomía y características del ID no abordable endoscópicamente.

La evaluación nutricional incluyó antropometría, composición corporal mediante bioimpedanciometría, determinación de catabolismo con nitrógeno total urinario, pre-albúmina y albúmina.

La RHI incluyó: a) Optimizar parámetros nutricionales previos al tratamiento quirúrgico para alcanzar cifras de albúmina mayor de $2,5 \mathrm{~g} /$ $\mathrm{dl}$ y un incremento mayor del 50\% del valor inicial de la pre-albúmina; b) Manejo dietético para maximizar el rendimiento del ID remanente con nutrientes específicos de acuerdo a la anatomía del tubo digestivo, suplementos vitamínicos, minerales y glutamina; c) Medicamentos para disminuir la motilidad intestinal y la secreción de ácido gástrico (loperamida, codeína, omeprazol); d) Administración de antibióticos para tratar la sobrepoblación bacteriana intestinal en los casos 
requeridos; e) Tratamiento o compensación de enfermedades concomitantes; $f$ ) Tratamiento de infecciones de la vía venosa central; g) Tratamiento quirúrgico, dentro de los cuales destacan; procedimientos para resolver fístulas entero-cutáneas, drenaje de abscesos abdominales crónicos, cierre de ostomías, recuperación de segmentos de colon o ID y elongación intestinal por medio de una enteroplastía transversa seriada (STEP), si fuera necesario ${ }^{15}$, además de la reparación simultánea de la pared abdominal por hernias incisionales, trayectos fistulosos y zonas de piel atrófica que cubrían las asas de intestino. Todas las decisiones de manejo médico/quirúrgico fueron discutidas en reuniones semanales de actualización de los pacientes.

El seguimiento fue realizado mediante controles clínicos periódicos hasta diciembre de 2015, evaluando la dependencia o no de NP, sea esta total o asociada a alimentación oral.

\section{Resultados}

La serie está compuesta por 14 pacientes con mediana de edad de 57 años (rango 26-84), 13 de ellos mujeres. Las causas de la II fueron; isquemia mesentérica en 7 pacientes; complicaciones quirúrgicas de resecciones segmentarias de ID o colon en 5 y la combinación de enteritis actínica y resección intestinal luego de radiación pelviana por cáncer ginecológico en 2 (Tabla 1). Al ingreso, 13 de ellos dependían de NPT, mientras que
1 paciente dependía de NP asociada a ingesta oral. Trece pacientes ingresaron con una ostomía (yeyunostomía, ileostomía o colostomía). Dos de ellos tenían además, fístulas entero-cutáneas de alto flujo. Las características demográficas, longitud del ID y colon remanente y tiempo de NPT previo ingreso al Programa de RHI, aparecen resumidos en la Tabla 2. La mediana de tiempo que los pacientes habían estado en NPT fue de 8 meses (rango 1 y 23), 4 de ellos con tiempo prolongado mayor de 1 año. La mediana de índice de masa corporal (IMC) al ingreso fue de 22,5 $\mathrm{kg} / \mathrm{mt}^{2}$ (rango 13-29) y de albúmina de $2,2 \mathrm{~g} / \mathrm{dl}$ (rango 1,8-3). Al egreso del programa la mediana de albúmina fue de 3,8 g/dl (rango 3,2-4,7).

La relación entre la longitud del ID y colon remanente, con los resultados de la RHI aparecen resumidos en la Tabla 2 . Nueve de los 14 pacientes, con resecciones extensas de ID, tenían además, resecciones segmentarias o de la totalidad del colon. De estos 9 pacientes, 6 presentaban ID remanente entre 50 y $100 \mathrm{~cm}$, asociado a la presencia de algunos segmentos o de la totalidad del colon. Dichos pacientes lograron la suspensión de la NP cuando se restableció quirúrgicamente la continuidad del tubo digestivo. En tanto que los otros 3 pacientes, accedieron al programa con $100 \mathrm{~cm}$ de ID, con una ostomía terminal, ausencia completa del colon y presencia de la totalidad del recto. En 2 de ellos fue posible la suspensión de la NP mediante la realización de una STEP (Figura 1), uno de ellos con anastomosis íleo-rectal concomitante (AIR). Estas STEP permitieron elongar el

Tabla 1. Causas de Insuficiencia Intestinal

\begin{tabular}{|c|c|c|c|}
\hline Causa & $\begin{array}{l}=14) \\
n\end{array}$ & Diagnóstico específico & \\
\hline \multirow[t]{3}{*}{ Isquemia } & 7 & Trombosis mesentérica & 5 \\
\hline & & Shock séptico, bajo débito & 1 \\
\hline & & Vólvulo de intestino delgado & 1 \\
\hline \multirow{4}{*}{$\begin{array}{l}\text { Complicaciones quirúrgicas de resecciones } \\
\text { intestinales }\end{array}$} & 5 & Tumor desmoides & 1 \\
\hline & & Bypass gástrico - Íleo mecánico & 2 \\
\hline & & Pseudomixoma peritoneal + QMT hipertérmica & 1 \\
\hline & & Resección GIST de YY - Fístula enterocutánea & 1 \\
\hline \multirow[t]{2}{*}{ Enteritis actínica } & 2 & Estrechez de anastomosis post-resección de ID & 1 \\
\hline & & Fibrosis y estrechez ileal & 1 \\
\hline
\end{tabular}

ID: Intestino delgado; YY: Yeyuno; QMT: Quimioterapia; GIST: Tumor del estroma gastrointestinal. 
Tabla 2. Características generales de la serie

\begin{tabular}{|c|c|c|c|c|c|c|}
\hline $\begin{array}{c}\text { Edad } \\
\text { (años) }\end{array}$ & Género & $\begin{array}{l}\text { ID } \\
\text { remanente } \\
(\mathbf{c m})\end{array}$ & $\begin{array}{c}\text { IG } \\
\text { remanente }\end{array}$ & Tratamiento quirúrgico & $\begin{array}{c}\text { Tiempo de NPT } \\
\text { pre-tratamiento } \\
\text { (meses) }\end{array}$ & $\begin{array}{l}\text { Estado } \\
\text { actual } \\
\text { de la NP }\end{array}$ \\
\hline 68 & $\mathrm{~F}$ & 50 & $\mathrm{Cl}+$ recto & Anastomosis yeyuno-cólica & 5 & $S$ \\
\hline 60 & $\mathrm{~F}$ & 60 & Completo & Anastomosis yeyuno-cólica & 2 & $S$ \\
\hline 26 & $\mathrm{~F}$ & 70 & $\mathrm{Cl}+$ recto & Enterorrafia & 10 & S \\
\hline 84 & $\mathrm{~F}$ & 90 & Completo & Anastomosis yeyuno-cólica & 6 & $S$ \\
\hline 38 & $\mathrm{~F}$ & 100 & Recto & Enteroplastía transversa seriada* & 8 & $S$ \\
\hline 57 & $\mathrm{~F}$ & 100 & Recto & Anastomosis íleo-rectal & 8 & $S$ \\
\hline 43 & $\mathrm{~F}$ & 100 & $\mathrm{Cl}+$ recto & Anastomosis colo-rectal & 23 & $S$ \\
\hline 65 & $\mathrm{~F}$ & 100 & Recto atrófico & Enteroplastía transversa seriada** & 15 & $S$ \\
\hline 65 & $\mathrm{~F}$ & 100 & Completo & Enterorrafia & 13 & $\mathrm{~S}$ \\
\hline 55 & $\mathrm{~F}$ & 120 & $\mathrm{CT}+\mathrm{Cl}+$ recto & Reversión bypass gástrico & 8 & $S$ \\
\hline 51 & $\mathrm{~F}$ & 130 & Completo & Anastomosis íleo-cólica & 3 & $S$ \\
\hline 67 & M & 130 & $\mathrm{CT}+\mathrm{Cl}+$ recto & Anastomosis yeyuno-cólica & 12 & $S$ \\
\hline 35 & $\mathrm{~F}$ & 150 & $\mathrm{CT}+\mathrm{Cl}+$ recto & Anastomosis yeyuno-cólica & 1 & $S$ \\
\hline 57 & $\mathrm{~F}$ & 200 & Completo & Anastomosis yeyuno-cólica & 20 & NP bisemana \\
\hline
\end{tabular}

${ }^{*}$ Con anastomosis íleo rectal; ${ }^{* *}$ Con enterorrafia. ID: Intestino delgado; IG: Intestino grueso; CT: Colon transverso; Cl: Colon izquierdo; NP: Nutrición parenteral; NPT: Nutrición parenteral total; S: NP suspendida.
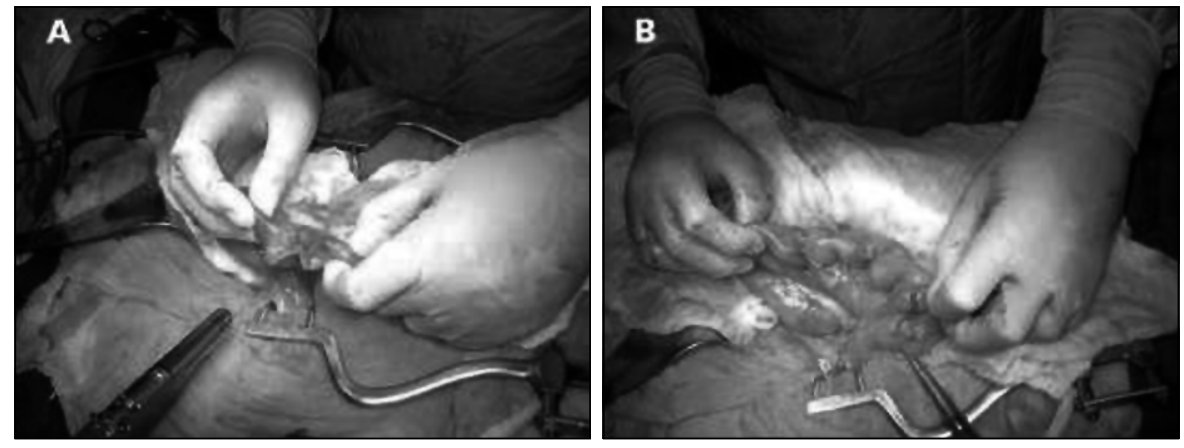

Figura 1. Enteroplastía transversa seriada.

ID remanente entre un 30 y $40 \%$ respectivamente. En el tercer paciente con esta misma condición $(100 \mathrm{~cm}$ de ID, ausencia de colon y presencia de recto), se practicó solamente la AIR porque el ID no había sufrido la dilatación necesaria para realizar una STEP. Los 5 pacientes restantes de la serie entraron al programa con un ID remanente entre 101 y $200 \mathrm{~cm}$, además de la conservación de la totalidad o al menos la mitad del colon. En 4 de ellos, el programa permitió suspender la NP luego de restablecer quirúrgicamente la continuidad del intestino. La única paciente en que no se logró este objetivo, fue debido a que el ID remanente tenía una extensa enteritis actínica. A pesar de que se intervino para resecar un segmento estrecho de íleon que le producía una obstrucción intestinal, sólo se logró eliminar la sintomatología obstructiva y disminuir la NP a un aporte bisemanal. Debido a sus condiciones generales no se consideró apta para el trasplante intestinal. En nuestra serie, no se 
registró mortalidad post-operatoria, ni tampoco existió daño hepático relevante mientras los pacientes estuvieron en NP.

Complicaciones de la vía venosa central fue observada en 6 pacientes, todas ellas infecciones tratadas con antibióticos y cambio de catéter. Los catéteres usados fueron: 4 catéteres definitivos (3 reservorios y 1 catéter central de inserción periférica). El resto correspondieron a catéteres subclavios.

En el postoperatorio, 2 pacientes presentaron celulitis en relación a la zona donde se ubicaba la ostomía previo a su cierre y 1 paciente desarrolló infección de la herida operatoria y una fístula entero-cutánea de bajo débito que cerró espontáneamente en el plazo de una semana. La mediana de hospitalización fue de 24 días (rango 4-110).

El seguimiento es completo y tuvo una mediana de 73 meses (rango 47-109). Dos pacientes fallecieron debido a patologías no relacionadas a su SIC y sin necesidad de NP. En 11 de los 12 pacientes restantes se logró la suspensión definitiva de la NP, manteniéndose esta condición en la actualidad. Por lo tanto, 13 de los 14 pacientes lograron la suspensión de la NP. La paciente en que no se logró la suspensión, pasó de una necesidad de NPT diaria a una NP bisemanal.

Los 12 pacientes de esta serie que sobreviven, desarrollan sus actividades habituales. Dos de ellos, con ID remanente de $100 \mathrm{~cm}$ y AIR, han necesitado hospitalizaciones transitorias por deshidratación secundarias a episodios de diarrea por trasgresión alimentaria. Uno de éstos, luego de 2 episodios de pancreatitis aguda por microlitiasis biliar fue sometido a papilotomía endoscópica retrógrada.

\section{Discusión}

La II es un concepto amplio, cuya causa más frecuente en adultos es el SIC por resecciones intestinales extensas. Publicaciones extranjeras en relación a la prevalencia de pacientes que dependan de NP domiciliaria, de la cual el SIC es la indicación más frecuente, sugieren que sería de aproximadamente entre 2 a 10 personas por millón/año ${ }^{2,3,16}$. En esta serie, aunque reducida, se expresan proporcionalmente las mismas causas de II por SIC en adultos que han sido comunicadas ${ }^{2,3,6}$.

El tratamiento de las enfermedades que ori- ginan un SIC, a menudo requieren de extensas resecciones de ID y colon, lo cual en la mayoría de los casos genera la necesidad de NP, ya sea en forma transitoria o permanente. En forma simultánea, se puede intervenir la dieta y agregar medicamentos para disminuir la motilidad intestinal y tratar la sobreproliferación bacteriana. De acuerdo con la respuesta a estas medidas, debe evaluarse la anatomía del intestino remanente para su reconstrucción autóloga, suprimiendo ostomías, reincorporando segmentos de intestino excluidos y practicando, si fuera necesario, procedimientos para elongarlo. Si estas medidas no logran la suspensión de la NP, el trasplante intestinal tiene una indicación precisa ${ }^{17-19}$.

Como se observa en este estudio la longitud del ID que se necesita para suspender la NP varía de acuerdo a los segmentos de intestino remanente (delgado y grueso). En este sentido, hubo 9 pacientes que presentando incluso $100 \mathrm{~cm}$ de ID remanente o menos, con presencia de segmentos de colon o sólo recto, lograron suspensión de la NP una vez que ingresaron al programa y se restituyó quirúrgicamente la continuidad del intestino. Es importante mencionar que en aquellos pacientes que teniendo menos de $100 \mathrm{~cm}$ de ID remanente y ausencia de colon, la STEP jugó un rol fundamental para lograr el desprendimiento de la NP. Esto grafica lo importante que es conservar el máximo de segmentos de colon en el tratamiento quirúrgico inicial (isquemia mesentérica, complicaciones quirúrgicas de resecciones intestinales o enteritis actínica), porque dicho colon remanente jugará un rol fundamental en la absorción de agua y electrolitos una vez que se restablezca quirúrgicamente la continuidad del tubo digestivo. Otros autores ${ }^{2-5,7}$, mencionan estas mismas observaciones destacando que en adultos con $100 \mathrm{~cm}$ de ID remanente o menos tendrían una alta probabilidad de NP permanente si hay ausencia de colon, mencionando además que en pacientes con SIC, la necesidad de NP dependerá de la longitud del ID remanente, pero también, del tipo de tratamiento quirúrgico ofrecido ${ }^{21}$.

La técnica de STEP fue comunicada el año 2003 como tratamiento del SIC en niños ${ }^{22}$. Sus resultados satisfactorios hicieron que fuera aceptada, extendiéndose su indicación progresivamente en adultos. Evaluaciones recientes comunican resultados satisfactorios, tanto que algunos autores consideran que es una técnica subutilizada. En 
una serie de 14 adultos con una mediana de ID remanente de $45 \mathrm{~cm}$, se logró un porcentaje medio de incremento de la longitud intestinal de $52 \%{ }^{23,24}$. Según estas observaciones sería prudente considerarla como indicada en pacientes que tengan menos de $100 \mathrm{cms}$ de ID remanente y ausencia o segmento muy cortos de colon.

Dentro de las posibles complicaciones descritas para la NPT destaca el daño hepático de diferente magnitud. En nuestra serie sólo destacaron alteraciones menores de las enzimas hepáticas, sin hiperbilirrubinemia, como ocurre con frecuencia en los pacientes que utilizan $\mathrm{NP}^{25-27}$. Creemos que se esto se relaciona al rápido inicio del aporte enteral en nuestros pacientes.

En la actualidad, la determinación de niveles plasmáticos de citrulina y la administración de un péptido análogo del glucagón (GLP-2), el primero para estimar la masa de enterocitos remanentes y el segundo para maximizar la capacidad absortiva, son elementos de apoyo en el tratamiento de pacientes con $\mathrm{II}^{28-30}$.

Otro factor en la II es el rol de la inmunidad innata y su relación con determinadas patologías inflamatorias intestinales, su gravedad y alteración en los mecanismos de normal AI. Esta inmunidad juega un rol fundamental en el equilibrio y reconocimiento de la flora normal y patógena dentro del intestino y en consecuencia de desencadenar o no una respuesta inmune inflamatoria ${ }^{31,32}$. Este complejo proceso se inicia en parte por receptores proteicos específicos a nivel intestinal, uno de ellos son los llamados dominios de oligomerización de nucleótidos (NOD2), presentes en macrófagos, células dendríticas y de Paneth ${ }^{33}$. Si bien se han descrito más de 30 mutaciones del gen NOD2, tres polimorfismos se asocian a Enfermedad de Crohn con fenotipo íleocecal estenosante y alteración en la AI posterior a la resección intestinal. También se ha descrito que este polimorfismo sería un factor de riesgo en pacientes sometidos a trasplante intestinal ${ }^{34,35}$.

En conclusión, aunque esta comunicación incluye un reducido número de pacientes, cobra valor teniendo en cuenta que la II por SIC es una condición poco frecuente en adultos, de alta complejidad médico quirúrgica y que los resultados han sido satisfactorios producto de un trabajo multidisciplinario, logrando la suspensión de la NP en prácticamente la totalidad de los pacientes.

\section{Referencias}

1. Dominique M. Intestinal Failure: Defenitions and Classifications. En: Alan N, Oliver G, Eamonn M, Kelly A, editores, Intestinal Failure. Malden, Massachusetts, USA: Editorial Blackwell Publishing; 2008. p. 57-65.

2. Tappenden KA. Pathophysiology of short bowel syndrome: Considerations of resected and residual anatomy. JPEN J Parenter Enteral Nutr 2014; 38 (1): 14-22.

3. Jeppesen PB. Spectrum of short bowel syndrome in adults: Intestinal insufficiency to intestinal failure. JPEN J Parenter Enteral Nutr 2014; 38 (1): 8-13.

4. Messing B, Crenn P, Beau P, Boutron-Ruault MC, Rambaud JC, Matuchansky C. Long-term survival and parenteral nutrition dependence in adult patients with the short bowel syndrome. Gastroenterology 1999; 117 (5): 1043-50.

5. Jeppesen PB, Mortensen PB. Significance of a preserved colon for parenteral energy requirements in patients receiving home parenteral nutrition. Scand J Gastroenterol 1998; 33 (12): 1175-9.

6. Adaba F, Rajendran A, Patel A, Cheung YK, Grant K, Vaizey SM, et al. Mesenteric Infarction: Clinical Outcomes After Restoration of Bowel Continuity. Ann Surg 2015; 262 (6): 1059-64.

7. Nightingale JM, Lennard-Jones JE, Gertner DJ, Wood SR, Bartram CI. Colonic preservation reduces need for parenteral therapy, increses incidence of renal stones, but does not change high prevalence of gallstones in patients with a short bowel. Gut 1992; 33 (11): 1493-7.

8. Dowling RH, Booth CC. Funtional compensation after small bowel resection in man. Lancet 1966; 2 (7455): 146-7.

9. Tappenden KA. Intestinal adaptation following resection. JPEN J Parenter Enteral Nutr 2014; 38 (1): 23-31.

10. Alberti R, Le Roy C, Cofré C, Patillo JC, Domínguez $\mathrm{B}$, Guerra JF. Advances in the management of children with intestinal failure. Rev Chil Pediatr 2014; 85 (2): 148-56.

11. Scolapio SJ, Fleming CR, Kelly DG, Wick DM, Zinsmeister AR. Survival of home parenteral nutrition-treated patients: 20 years of experience at the Mayo Clinic. Mayo Clin Proc 1999; 74 (3): 217-22.

12. Messing B, Lemann M, Landais P, Gouttebel MC, Gérard-Boncompain M, Saudin F, et al. Prognosis of patients with nonmalignant chronic intestinal failure receiving long-term home parenteral nutrition. Gastroenterology 1995; 108 (4): 1005-10.

13. Sudan D, DiBaise J, Torres C, Thompson J, Reynor 
S, Gilroy R, et al. A multidisciplinary approach to the treatment of intestinal failure. J Gastrointest Surg 2005; 9 (2): 165-76.

14. Abu-Elmagd K, Bond G, Matarese L, Costa G, Koritsky $\mathrm{D}$, Laughlin $\mathrm{K}$, et al. Gut rehabilitation and intestinal transplantation. Therapy 2005; 2 (6): 853-64.

15. Thompson J, Sudan D. Intestinal lengthening for short bowel syndrome. Adv Surg 2008; 42 (1): 49-61.

16. Buchman AL. Etiology and initial management of short bowel syndrome. Gastroenterology 2006; 130 (2): 5-15.

17. Fishbein TM. Intestinal transplantation. N Engl J Med 2009; 361 (10): 998-1008.

18. Fishbein T, Matsumoto CS. Intestinal replacement therapy: Timing and indications for referral of patients to an intestinal rehabilitation and transplant program. Gastroenterology 2006; 130 (2): 147-51.

19. Mercer DF, Iverson AK, Culwell KA. Nutrition and small bowel transplantation. Nutr Clin Pract 2014; 29 (5): 615-20.

20. Wilmore DW, Byrne TA, Persinger RL. Short bowel syndrome: new therapeutic approaches. Curr Probl Surg 1997; 34 (5): 389-444.

21. Allard JP, Jeejeebhoy KN. Nutritional support and therapy in the short bowel syndrome. Gastroenterol Clin North Am 1989; 18 (3): 589-601.

22. Kim HB, Fauza D, Garza J, Oh JT, Nurko S, Jaksic T. Serial transverse enteroplasty (STEP): A novel bowel length. J Pediatr Surg 2003; 38 (3): 425-9.

23. Bellolio F, Klaassen J, Pulgar D, Molina ME, Pinedo G, Zúñiga A. Serial transverse enteroplasty for short bowel syndrome. Case report. Rev Med Chile 2010; 138 (4): 478-82.

24. Yannam GR, Sudan DL, Grant W, Botha J, Langnas A, Thompson JS. Intestinal lengthening in adult patients with short bowel syndrome. J Gastrointest Surg 2010; 14 (12): 1931-6.

25. Howard L, Ashley C. Management of complications in patients with receiving home parenteral nutrition. Gastroenterology 2003; 124 (6): 1651-61.

26. Salvino R, Ghanta R, Seidner DL, Mascha E, Xu Y,
Syeiger E. Liver failure is uncommon in adults receiving long-term parenteral nutrition. JPEN J Parenter Enteral Nutr 2006; 30 (3): 202-8.

27. Luman W, Shaffer JL. Prevalence, outcome and associated factors of deranged liver funtion test in patients on home parenteral nutrition. Clin Nutr 2002; 21 (4): 337-43.

28. Luo M, Fernández-Estívariz C, Manatunga A, Bazargan $\mathrm{N}, \mathrm{Gu} \mathrm{L}$, Jones D, et al. Are plasma citrulline and glutamine biomarkers of intestinal absorptive function in patients with short bowel syndrome? J Parenter Enteral Nutr 2007; 31 (1): 1-7.

29. Jiangfeng G, Weiming Z, Ning L, Fangnan L, Li T, Nan $\mathrm{L}$, et al. Serum citrulline is a simple quantitative marker for small intestinal enterocytes mass and absorption function in short bowel patients. J Surg Res 2005; 127 (2): 177-82.

30. Jeppesen PB, Pertkiewicz M, Messing B, Iyer K, Seidner DL, O'keefe SJ, et al. Teduglutide reduces need for parenteral support among patients with short bowel syndrome with intestinal failure. Gastroenterology 2012; 143 (6): 1473-81.

31. Zasloff M. Antimicrobial peptides in health and disease. N Engl J Med 2002; 347 (15): 1199-200.

32. Magalhaes JG, Tattoli I, Girardi SE. The intestinal epithelial barrier: how to distinguish between the microbial flora and pathogens. Semin Immunol 2007; 19 (2): 10615.

33. Strober W, Murray PJ, Kitani A, Watanabe T. Signalling pathways and molecular interactions of NOD1 and NOD2. Nat Rev Immunol 2006; 6 (1): 9-20.

34. Guerra JF, Zasloff M, Lough D, Abdo J, Hawksworth J, Mastumoto C, et al. Nucleotide oligomerization domain 2 polymorphisms in patients with intestinal failure. $\mathrm{J}$ Gastroenterol Hepatol 2013; 28 (2): 309-13.

35. Economou M, Trikalinos TA, Loizou KT, Tsianos EV, Loannidis JP. Differential effects of NOD2 variants on Crohn`s disease risk and phenotype in diverse populations: a metaanalysis. Am J Gastroenterol 2004; 99 (12): 2393-404. 